\title{
Numerical Model for Biaxial Earthquake Response of Reinforced Concrete
}

\author{
Cemalettin Dönmez* \\ Civil Engineering Department, Izmir Institute of Technology, Urla, Izmir 35430, Turkey
}

$\&$

Mete A. Sözen

Kettelhut Distinguished Professor of Structural Engineering, School of Civil Engineering, 550 Stadium Mall Drive, West Lafayette, IN 47907-2051

\begin{abstract}
A numerical constitutive model is developed to simulate the biaxial nonlinear flexural response of slender reinforced concrete members subjected to earthquake excitation. The model is tested using data from two types of experiments with reinforced concrete elements: (1) elements subjected to varying pseudo-static biaxial lateral loads and (2) elements that responded biaxially to simulated earthquake motions. The goal for the model was not only to help determine the absolute maxima for earthquake response but also to enable calculation of the entire waveform, including the ranges of low-and moderateamplitude response. The comparisons of measured and calculated results and sensitivity of the proposed model to variations in the input parameters are discussed. The output was found to be insensitive to the changes in input parameters related to concrete and sensitive to input parameters related to reinforcing steel. The results of the calculations were tested using experimental data.
\end{abstract}

\section{INTRODUCTION}

This article describes a numerical model for nonlinear biaxial response analysis of reinforced concrete members subjected to strong ground motion in one or two hori-

*To whom correspondence should be addressed. E-mail: sozen@ purdue.edu. zontal directions. Displacement response of reinforced concrete to forces that vary randomly in direction and magnitude results from a complex interaction of the properties of the concrete and the steel and the bond between them. It is optimistic to expect event reproducibility. Identical loading histories imposed on identical elements are unlikely to lead to identical displacement responses. The model has been designed with a view to balance the quality of the output against the number of assumptions required for its implementation. The force-displacement relationships for both concrete and steel were initially based on the simplest assumptions necessary to model nonlinear response. These assumptions were modified only if they produced results that were unacceptable in relation to experimental results and the reason for the discrepancy was understood in terms of material properties.

In the numerical model, the reinforced concrete section is represented by a set of nonlinear springs with appropriate material properties (Figure 1). This approach demands considerable computational time but provides convenience and flexibility. It also leads to time savings in understanding its operation and in its implementation. Zeris (1986), Sfakianis and Fardis (1991), and Ricles et al. (1998) have used this technique. Crack opening, crack closing, softening of concrete, the Bauschinger effect, unloading and reloading 


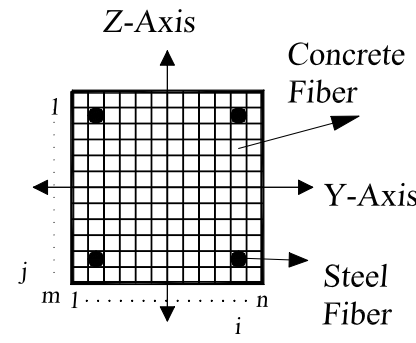

(a) Discretization of a rectangular section

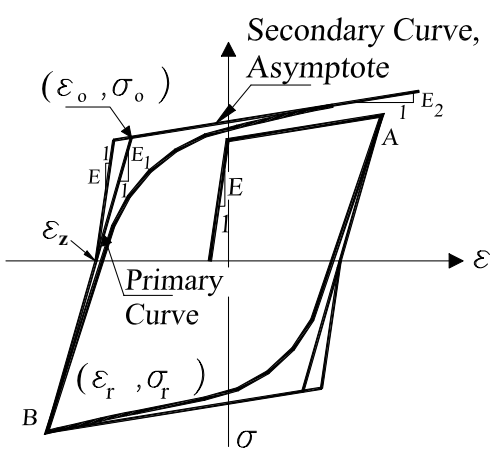

(b) Reinforcing steel hysteresis model

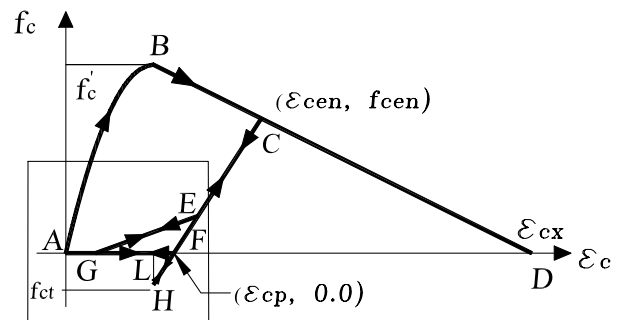

$\prod$ (c) Concrete hysteresis model

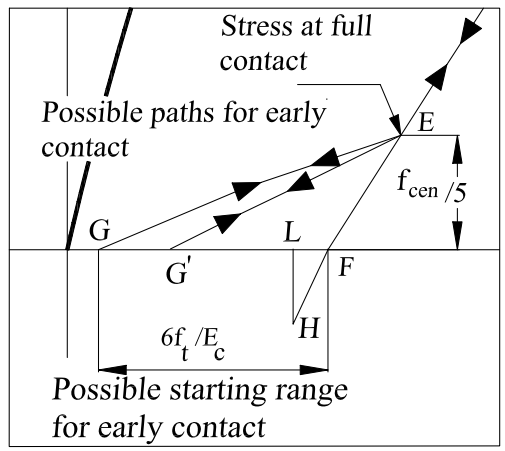

(d) Rules for defining "incomplete contact" of concrete at a cracked section

Fig. 1. Representation of the reinforced concrete section by a set of nonlinear springs.

of the reinforcing steel, and changes in the length of the plastic hinge region along the member can all be modeled conveniently.

Even though the model is inherently capable of simulating constant or variable axial load, in this study the cases considered involved flexure only. It is assumed that the element has sufficient transverse reinforcement to maintain its strength under stress reversals (Pujol, 2002). The results of the model have been tested using measurements obtained in static and dynamic tests. The range of concrete strength covered by the data was from 20 to $48 \mathrm{MPa}$. Yield stress of steel varied from 410 to $470 \mathrm{MPa}$.

\section{MATERIAL MODELS}

Concrete in compression was represented by the Ritter parabola (Path A-B, Figure 1c) and a linear extension (Path B-D, Figure 1c), after Hognestad (1951). The unloading path was defined to be linear (Path C-F, Figure 1c) with its slope based on the observations by Karsan and Jirsa (1969). Initially, reloading was set to follow the unloading path. Pilot studies indicated that this approach failed to simulate the observed response. Compressive stress build-up associated with crack closing was not a simple phenomenon to be captured by using the same path in reloading as the one that had been used in unloading. The reloading (compressive loading after cracking) slope was modified to recognize closing of the crack (re-establishment of contact between the boundaries of the crack) at a virtual strain lower than that at which the unloading path crossed the strain axis (Path GE, Figure 1d).

Reinforcement was initially modeled by bilinear curves in tension and compression with three parameters defining the initial and secondary slopes and the yield stress. When it was observed that the hysteresis based on the bilinear curve failed to simulate energy dissipation at low amplitudes, a different model based on the Menegotto-Pinto equation (Menegotto and Pinto, 1973; [M-P]) was adopted. The steel hysteresis model with M-P curves is illustrated in Figure 1b. Detailed explanations of the material models can be found in Appendix A and in Dönmez (1998).

\section{MOMENT-DEFLECTION RELATIONSHIPS}

The nonlinear and path dependent character of the assumed hysteresis relationships for the two materials necessitated an incremental and iterative algorithm. The algorithm described here is used to calculate the 


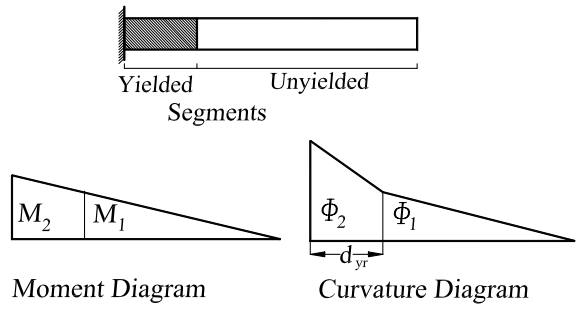

(a) Member segments and the assumed curvature distribution along its length

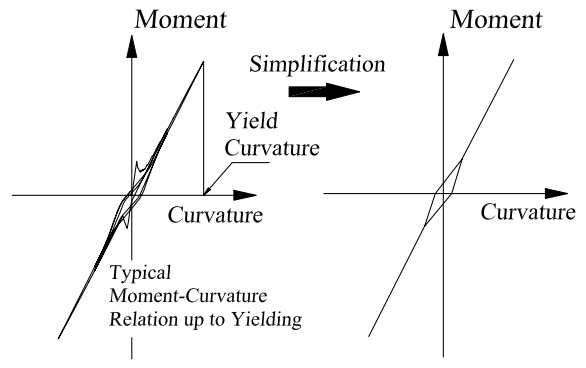

(c) Simplified relation representing $M-\phi$ relation after cracking up to yielding

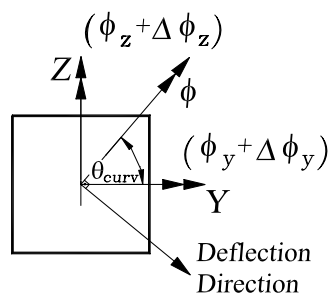

(b) Components of the curvature vector

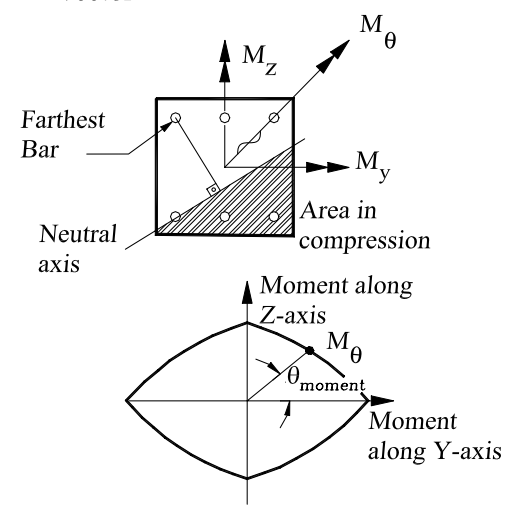

(d) Components of the moment vector and the moment interaction curve at yielding

Fig. 2. Necessary definitions for the calculation of the incremental deflections for the given incremental moments.

moment increments in a reinforced concrete cantilever for given deflection increments at its free end. Member deflection is considered to comprise three components: deflections attributed to (1) flexure, (2) shear, and (3) slip of the reinforcing bar. The flexural component is determined using the curvature distribution along the span of the "cantilever member" shown in Figure 2a. Curvature distribution along the span is assumed to vary at different rates in the (1) yielded segment and (2) the unyielded segment. The relationship of moment to curvature at the section of maximum moment, $\mathrm{M}_{2}$ and $\phi_{2}$, is determined by the hysteresis model (see Appendix B for details). If the moment $\mathrm{M}_{2}$ at the base is more than the yield moment, the location of the yield moment, $\mathrm{M}_{1}$, in the span is determined directly from statics. The curvature, $\emptyset_{1}$, denotes the yield curvature in Figure $2 \mathrm{a}$. If $\mathrm{M}_{2}$ increases, the location of $\mathrm{M}_{1}$ moves toward the free end of the cantilever. If $\mathrm{M}_{2}$ decreases (after having exceeded the yield moment), the "yield region," the distance between the locations of the moments $\mathrm{M}_{1}$ and $\mathrm{M}_{2}$ is not reduced but the curvature $\phi_{1}$ is reduced. The "yield region" length remains unchanged at this stage because of the disintegration of the materials from their pristine condition. The simplest possible assumption about the variation of the curvature within the region is made and curvature is assumed to vary linearly. If the moment $\mathrm{M}_{1}$ is reduced so that the cracks start to close, the change in curvature depends on the level of contact and follows the simplified relation representing the $\mathrm{M}-\varnothing$ relation after cracking up to yield (Figure 2c). A representative summary of the assumed curvature distributions is given in Figure 3 at different stages of loading.

The slip of the reinforcing bar is estimated by assuming a constant development length of 50 bar diameters for materially coupled and 40 bar diameters for materially uncoupled members and a uniform bond stress. Materially coupled refers to interaction between the bending stresses resulting from loading in orthogonal directions. The slip is calculated for the reinforcing bar that has the highest strain in the section. The ratio of the calculated slip to the distance of the bar from the neutral axis is assumed to be the rotation caused by slip, $\theta_{\mathrm{s}}$. The deflection component related to slip is obtained as the product of the rotation $\theta_{\mathrm{s}}$ and the length of the member.

The shear deflection was calculated assuming a linear, uncracked reinforced concrete member.

The main difficulty in calculating the incremental moments for the given incremental deflections of the nonlinear and path dependent moment-deflection relations arises in finding the appropriate curvature increments. These are determined by the iterative procedure explained in Appendix B. 


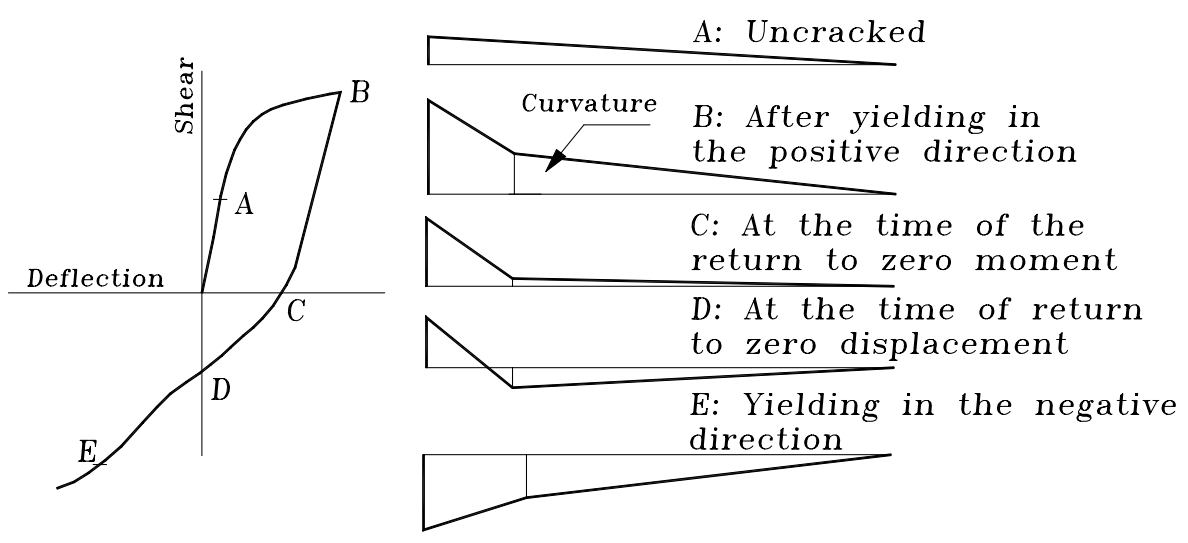

Fig. 3. Representative summary of the assumed curvature distribution.

\section{SENSITIVITY ANALYSIS}

A sensitivity study was performed to define the needed accuracy of the input parameters for applications of the model. The effects on displacement history were evaluated for the parameter ranges listed in Table 1 . The term "consistent definition" in the table refers to the determination of the "yield region" consistently by defining it to extend from the section where the yield moment is encountered to the section of maximum moment.

The structural element assumed for the sensitivity study was a cantilever supporting a concentrated weightless mass subjected at its base to horizontal components of the strong-motion record obtained in 1940 in El Centro, California (California Institute of Technology, 1971). The record was scaled to have a maximum acceleration of $0.5 \mathrm{~g}$ for the north-south component. The east-west component was scaled to keep the original ratio of the two components. The dimensions and the reinforcement details of the cantilever are presented in Figure 4. The nominal compressive strength of the concrete and yield stress of the steel assumed for the system were $41.4 \mathrm{MPa}(6,000$ psi) and $414 \mathrm{MPa}(60,000 \mathrm{psi})$, respectively. The concentrated mass at the tip was $6,130 \mathrm{~kg}$. Its initial period was
0.25 seconds. The system reached a maximum calculated drift ratio of approximately four times the drift at yield.

Sensitivity of the proposed model was considered in terms of maximum displacements and the waveform of the displacement histories. A brief summary of the results will be provided below. Further information can be found in Dönmez (1998). Based on response sensitivity, the input parameters considered can be divided into two groups.

1. The output of the model was found to be sensitive to changes in: (i) the yield stress of the reinforcing steel; (ii) the transition parameter of the Menegotto-Pinto model; and (iii) development length of the reinforcing bars. Variation of transition parameter of the Menegotto-Pinto model also affected the damping characteristics of the response. The changes of the absolute-maximum displacements were less than $19 \%$.

2. The output of the model in phases of low response (low response is characterized as having a drift less than half of the drift at yield) was found to be sensitive to: (i) concrete strength; (ii) parameters defining crack closing; (iii) initial modulus of elasticity for the concrete; and (iv) the definition of the "yield

Table 1

Parameters chosen for the sensitivity analysis

\begin{tabular}{lll}
\hline Parameters & Reference response parameters & Variation \\
\hline Concrete strength & $f_{c}^{\prime}$ & $0.8 f_{c}^{\prime}$ and $1.2 f_{c}^{\prime}$ \\
Parameters defining "early contact" of concrete & $6,0.2$ & No "Early Contact" and $9,0.4$ \\
Initial modulus of elasticity of the concrete & $4,700\left(f_{c}^{\prime}\right)^{0.5}$ & $4,150\left(f_{c}^{\prime}\right)^{0.5}$ and $5,300\left(f_{c}^{\prime}\right)^{0.5}$ \\
Yield stress of the reinforcing steel & $f_{y}$ & $0.8 f_{y}$ and $1.2 f_{y}$ \\
Transition parameter of Menegotto-Pinto model & 17 & 12 and 25 \\
Length of "yield region" & Consistent definition & $0.5^{*}($ Consistent definition $)$ \\
Bar development length & 50 bar diameters & 40 bar dia. and 60 bar dia. \\
\hline
\end{tabular}




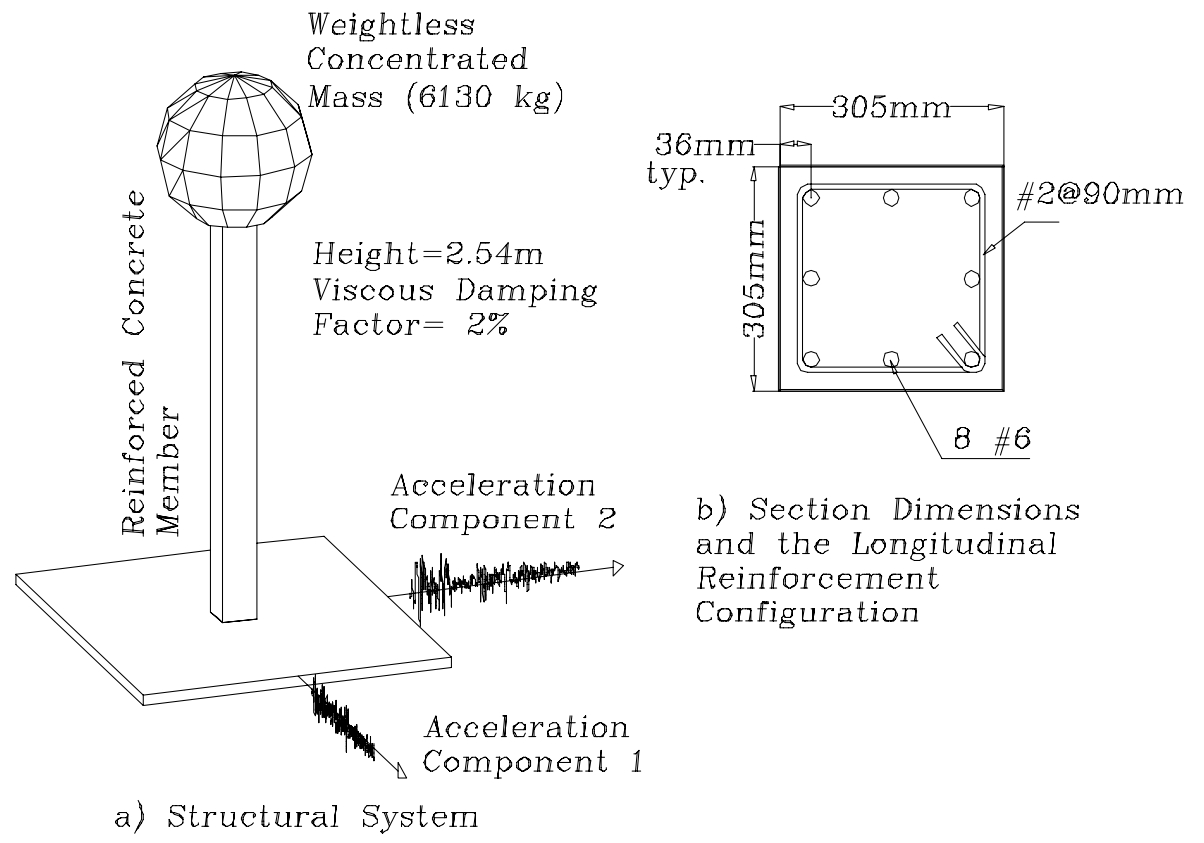

Fig. 4. The dimensions and the reinforcement details of the system used in sensitivity analysis.

region" along the column. Changes of the absolutemaximum displacements were less than $3 \%$.

Typical response sensitivities for the groups mentioned above are illustrated for the effect of change in yield stress of reinforcing steel in Figure 5a and for the effect of change in concrete strength in Figure 5b. The sensitivity of calculated response to changes in the first group of input parameters was strong for maximumdisplacement, waveform, and periodicity. The effect of the second group of input parameters was weak and limited to the low-response phases. It is clear that accurate knowledge of concrete strength is not important for getting satisfactory estimates of the displacement response at drifts beyond half the drift at yield. On the basis of the studies made for the sensitivity of the proposed model, if there is an error of $20 \%$ in the estimate of the concrete strength, it would affect the calculated maximum displacement by less than $3 \%$ with negligible changes in waveform and periodicity. On the other hand, an error of $20 \%$ in the estimate of the yield stress of reinforcing steel would change the displacement response by $15 \%$ with notable changes in the waveform and periodicity. The choices for the first group of parameters have to be considered carefully in assembling a model for calculating response.

\section{COMPARISONS OF EXPERIMENTAL AND CALCULATED RESULTS}

The experimental results by Otani and Cheung (1981) were used for testing the proposed hysteresis algorithm in the static domain. In the Otani-Cheung tests, reinforced concrete cantilevers were subjected to uniaxial and biaxial bending by application of static forces. The experimental results by Dragovich (1996) were used for testing the proposed algorithm in the dynamic domain. Dragovich subjected reinforced concrete cantilevers to uniaxial and biaxial bending dynamically (base excitation). In both loading cases, there were no axial loads on the cantilevers.

\subsection{Statically loaded specimens}

Data from the Otani-Cheung specimens 5 and 7 were used for testing the proposed model. The dimensions and the reinforcing steel configuration of the chosen specimens are given in Figure 6. Specimen 5 was tested under uniaxial loading and specimen 7 was tested under biaxial loading. The displacement paths that the specimens were forced to follow are presented in Figures $7 \mathrm{a}$ and d. The longitudinal bars of the specimens were welded to a grid of steel plates at the base at a distance of 20 bar diameters from the face of the joint. Numerical modeling of the test stopped at a rather large drift ratio of $6 \%$.

The measured and the calculated force-displacement relations of Specimen 5 are presented in Figures $7 \mathrm{~b}$ and c. To facilitate visual comparison, responses in different drift-ratio ranges are shown by different line types. Up to a drift ratio of $1.3 \%$, the curve is represented by a thin broken line. From $1.3 \%$ to $3.0 \%$, the line is solid. From $3.0 \%$ to $6.0 \%$, it is broken but thicker. 


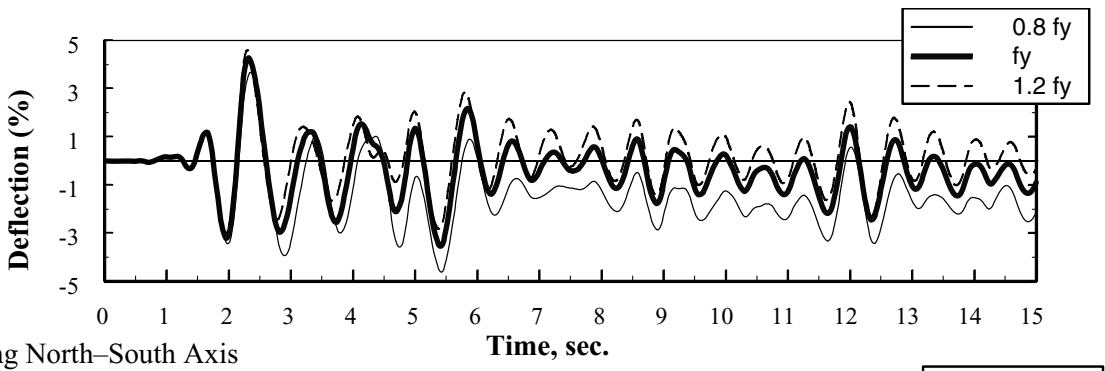

Along North-South Axis

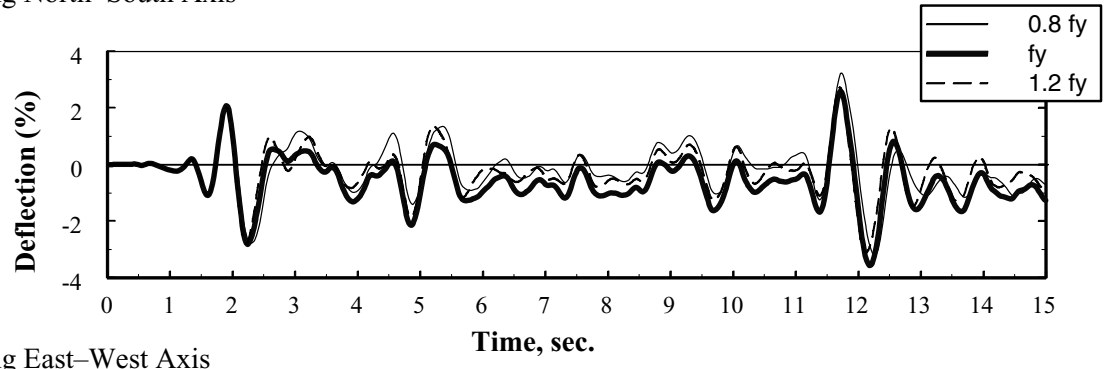

Along East-West Axis

(a) Effect of Change in Reinforcing Bar Yield Stress

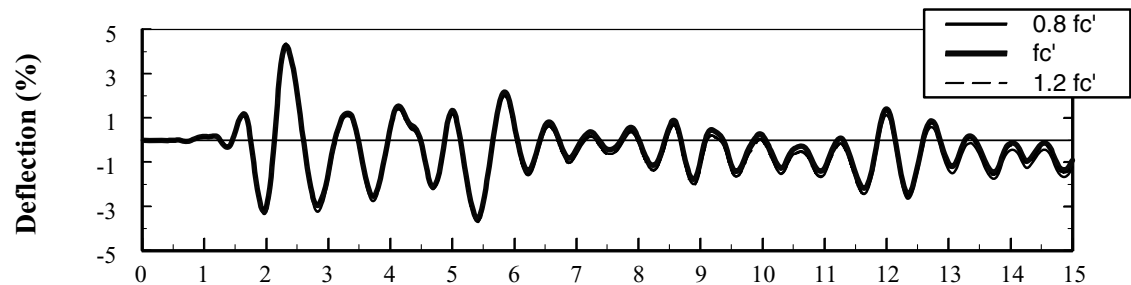

Along North-South Axis

Time, sec.

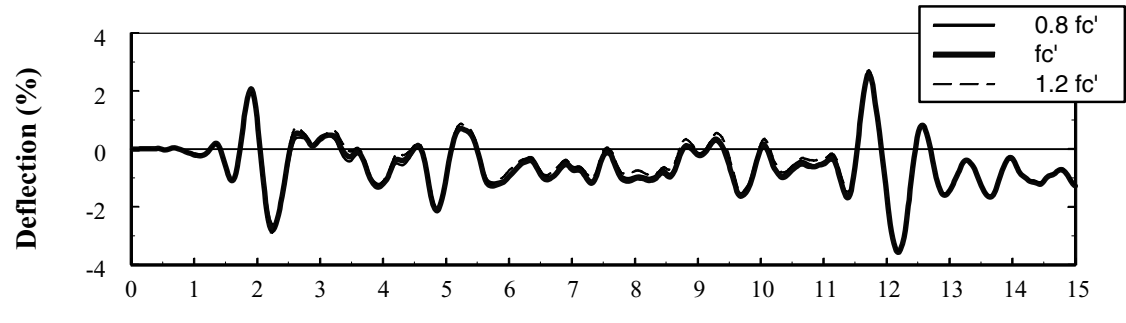

Along East-West Axis

Time, sec.

(b) Effect of Change in Concrete Strength

Fig. 5. Representative examples of displacement histories for the changes in parameter groups defined.

Comparison of the data in Figure 7 is shown primarily to demonstrate that the model, designed for biaxial loading, provides a credible representation of hysteretic response if the action is uniaxial. The comparison of measured and calculated mean stiffnesses (defined by the slope of a line drawn to extreme points on the hysteresis loops) were excellent at drift ratios up to $1.3 \%$. In this range, the computed and measured areas within the hysteresis curve did not compare well because of the sensitivity of the total area, that was small, to small changes in the defining curves. At drift ratios over $1.3 \%$ the computed energy dissipation and mean stiffness had maximum errors of $15 \%$ and $10 \%$, respectively.

The loading history imposed on Specimen 7 is shown in Figure $7 \mathrm{~d}$. The top of the specimen was forced to travel in a horizontal plane in one direction at a time so that the travel path was essentially "square" at each level of applied displacement. The comparison of measured and calculated force-displacement relationships was stopped at a drift ratio of $4 \%$.

The measured and the calculated force-displacement relations for Specimen 7, east-west (EW) direction, are 


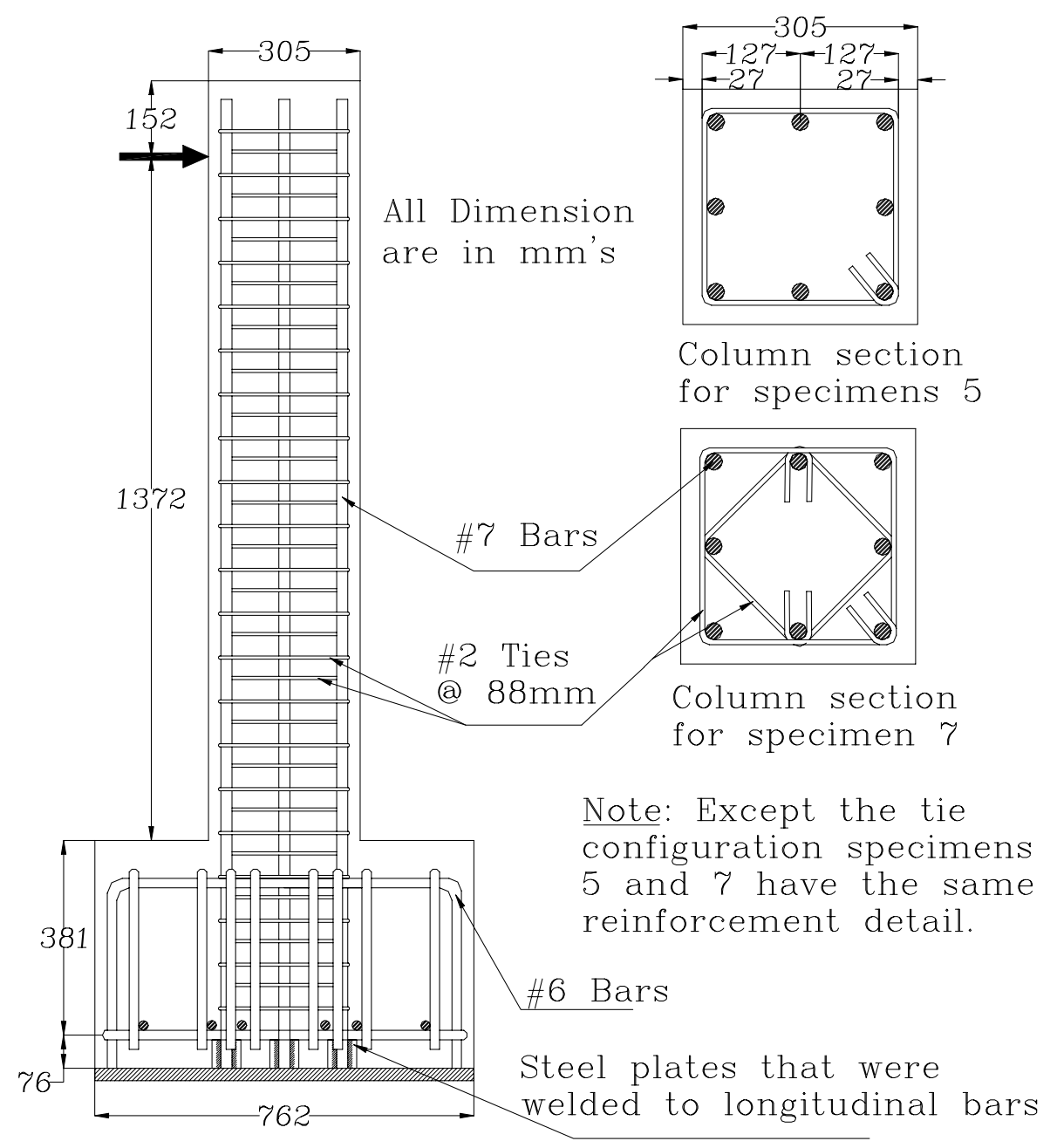

Fig. 6. Arrangement of reinforcement in specimens 5 and 7, tested by Otani and Cheung (1981).

presented in Figures 7e and f. Response is separated into three groups on the basis of drift ratio attained: approximately $0.5 \%, 1.3 \%$, and $2.5 \%$. For each group peak-force levels were in error by less than $\pm 10 \%$. Except for the first group, the energy dissipation of the proposed model was within $\pm 15 \%$ of the measurements. The calculated stiffnesses for the first two groups were within $\pm 6 \%$ of those measured. For the third group, the error increased up to $17 \%$ because of the insensitivity of the material model to the effects of confinement of concrete.

Several times through the loading history, the top deflection of Specimen 7 was kept at a certain level in one direction and was increased in the orthogonal direction. Because of this loading scheme, sudden drops were observed for the moment in the direction in which the displacement was kept constant (Figures 7e and f). The proposed algorithm response is sensitive to this phenomenon and indicated similar drops in moment.
The bidirectional resistance trace for both the measured and calculated responses are presented in Figure 8. The calculated response was comparable to the measured response. Otani and Cheung (1981) mentioned that the extreme traces in the north-west quadrant of Figure 8 represented the limits of resistance of the column. The calculated traces exhibit a similar behavior at the same locations defining the "yield surface."

\subsection{Dynamically loaded specimens}

The purpose of Dragovich's experiments (1996) was to study the effects of the combined torsionaltranslational loading in reinforced concrete. The torsional-translational response was considered in terms of dynamic and material coupling. A conceptual model of the test specimens is given in Figure 9a. As illustrated in Figure 9a, the base motion along the $X$-axis generates moments about $\mathrm{Y}$ and $\mathrm{Z}$-axes, because of the 


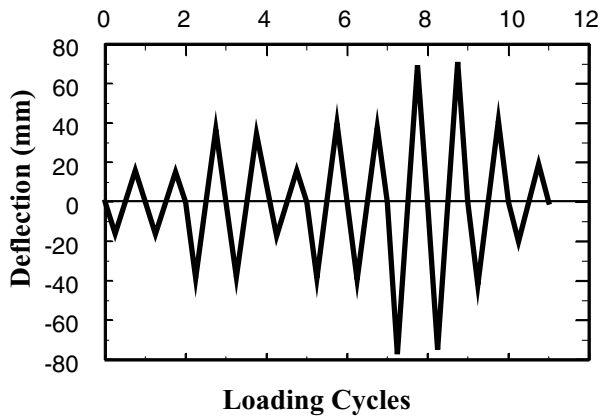

(a) Displacement path for specimen 5

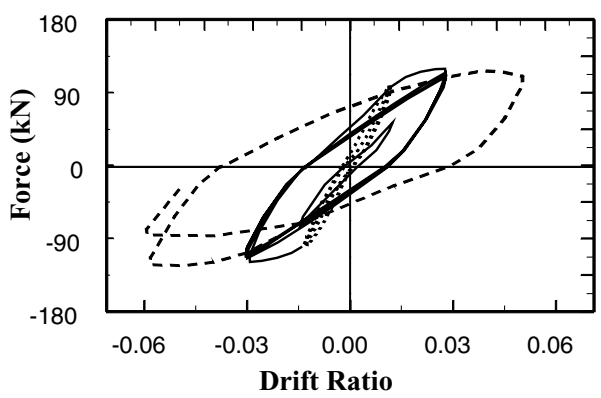

(b) Measured force-disp. relation for spec. 5

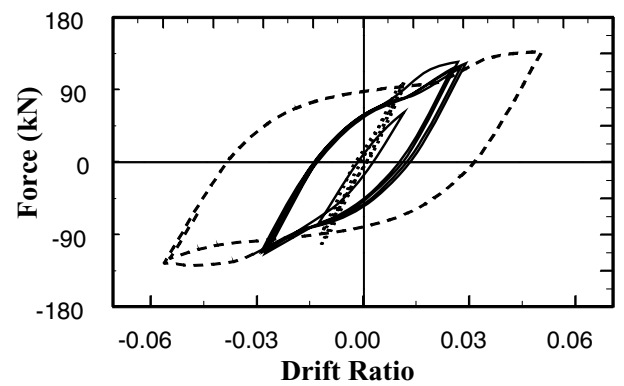

(c) Calculated force-disp. relation for spec. 5

Specimen 5

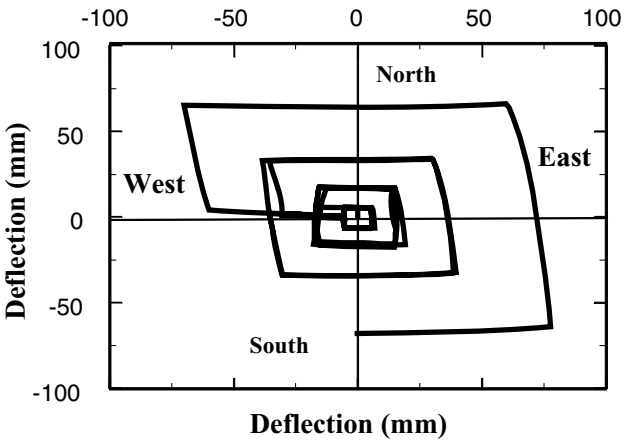

(d) Displacement path for specimen 7

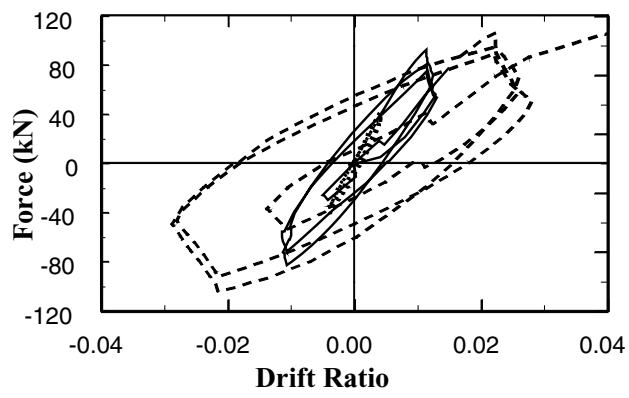

(e) Measured force-disp. relation for spec. 7

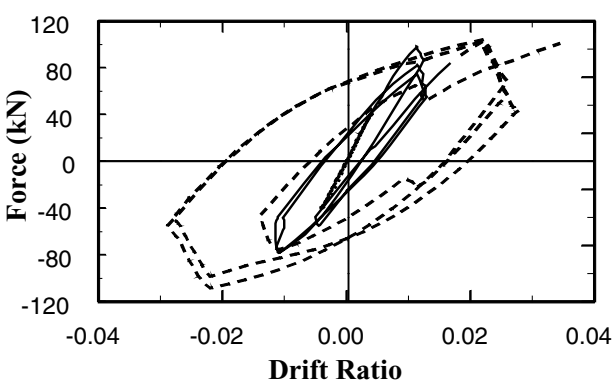

(f) Calculated force-disp. relation for spec. 7

Specimen 7 EW Component

Fig. 7. The displacement paths that the specimens were forced to follow and the measured and the calculated force-displacement relations for Otani's specimens.

eccentricity e, of the mass m. Configuration of the members dictates the material coupling. Dragovich used two different member configurations in the experiments. The first configuration caused only dynamic coupling. As illustrated in Figure 9b, the moment along the Y-axis was resisted by the lower beam, and the moment along the $\mathrm{Z}$-axis was resisted by the upper beam. Since each beam resisted moments along one axis, there was no material coupling. The second configuration involved both dynamic and material coupling. Moments about axes $\mathrm{X}$ and $\mathrm{Z}$ were resisted by a single beam (Figure 9c). This beam was subjected to randomly varying biaxial bending.
The specimens were designed to observe the effect of changes in three parameters: strength, mass distribution, and material coupling. Specimen strength was varied by changing the amount of longitudinal reinforcement in the beam and the mass distribution was varied by changing the mass eccentricity along the Y-axis (Figure 9a). Two values of the mass distribution and two values of the beam section strength were used both for the materially coupled and for the materially uncoupled specimens. A total of eight specimens were tested.

The north-south component of the 1940 El Centro earthquake record (California Institute of Technology, 1971) was used as the base motion. The time scale was 

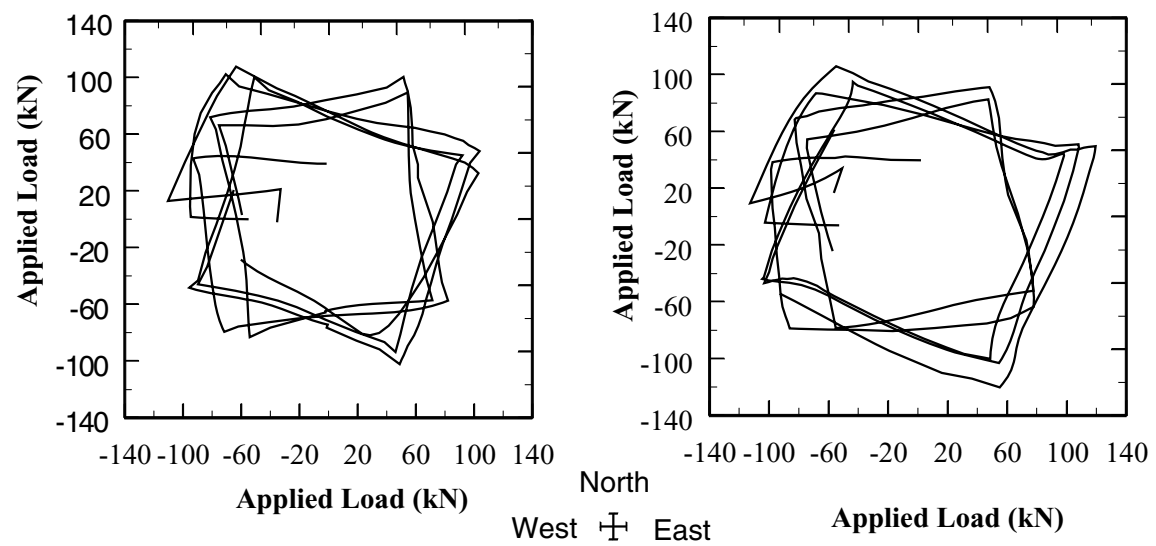

Measured

South

Calculated

Fig. 8. Measured and calculated biaxial moment traces of specimen 7 .

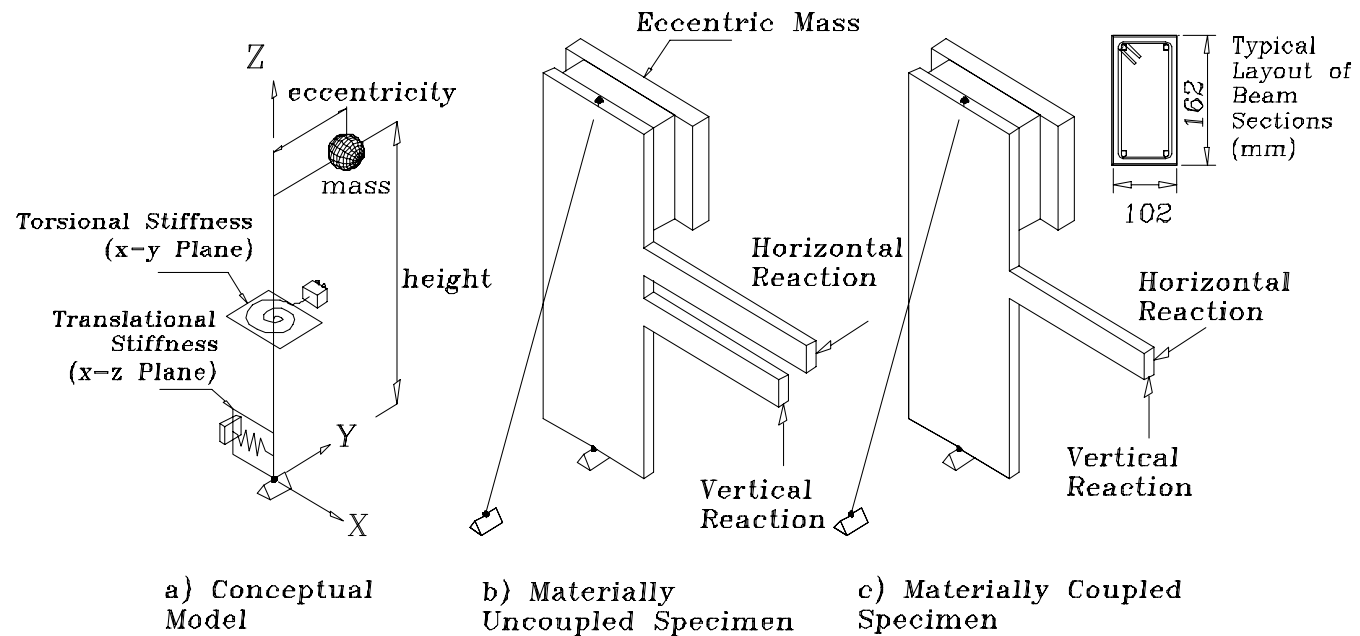

Fig. 9. Dragovich's specimens (1996).

compressed by a factor of two, to shift the region of nearly constant linear velocity response closer to the frequencies of the test specimens and to keep the deflections of the earthquake simulator within its operational limits. Each test specimen was subjected to three earthquake simulations of increasing intensity to produce successively increasing levels of damage. The demand for the first set of simulations was low. The specimen responses were below yield level. The second sets of simulations were used for comparisons with the calculated simulations of the proposed model. During those tests the members of the specimens reached drift ratios of $1 \%$ to $3 \%$. Comparisons are made in terms of maximum rotation, rotation-history (waveforms), and the moment-rotation hysteresis.

Measured and calculated rotation histories for the selected materially coupled and uncoupled specimens are presented in Figure 10. The mean ratio of the measured to calculated absolute-maximum rotations for the coupled specimens was 0.96. For the uncoupled specimens, it was 0.90 . Rotation histories of the specimens exhibited phases of high and low responses. The agreement of measured and calculated curves was highly satisfactory for the high response phases (high response is characterized as having a drift more than half of the drift at yield). For the low response phases, the agreement was not as good. At low response especially after yielding, the calculated rotations were sensitive to the assumed material response. This response can be observed in the 4-6 second and the 7-9 second intervals of the Y-axis response in Figure 10b. The mean ratio of the measured and calculated absolute-maximum moments was 1.05 for the coupled specimens and 1.12 for the uncoupled specimens. The difference between the measured and the calculated values were primarily attributed to strain hardening and the strain-rate effects of the reinforcing bar. 

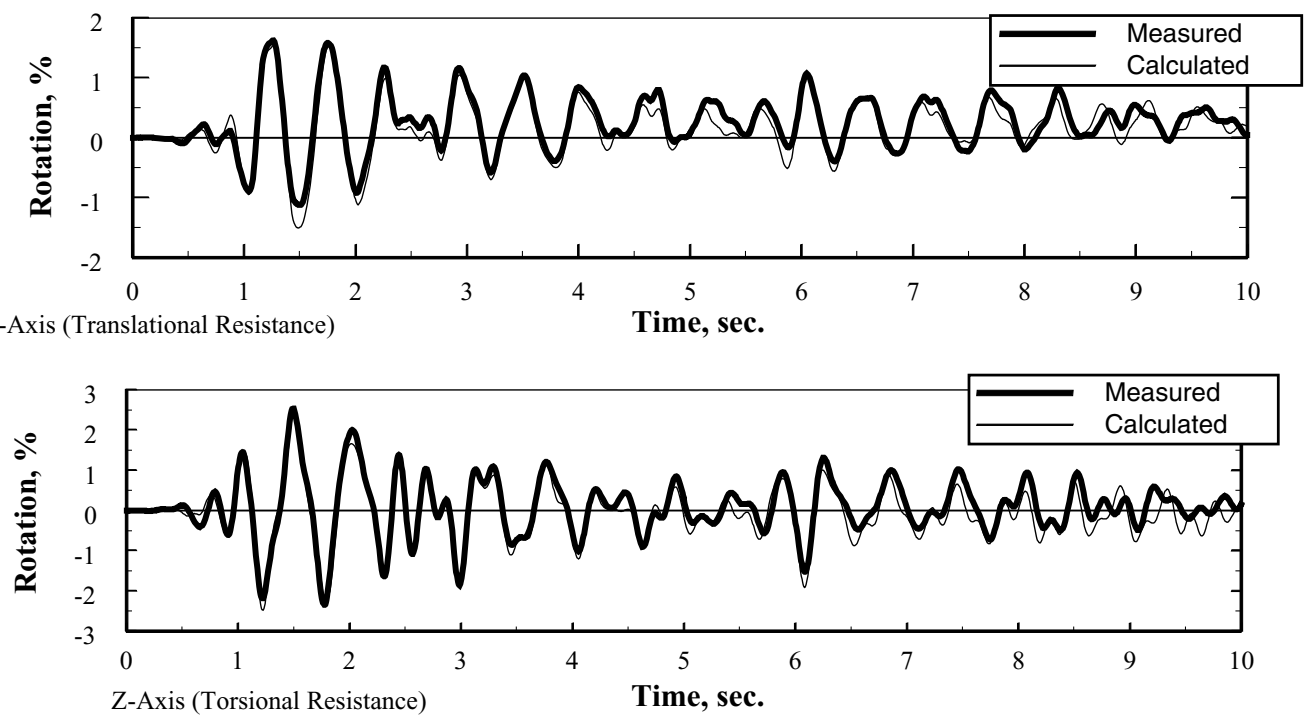

a) Displacement Histories for Rotation About Y and Z Axes,

Specimen CLW (coupled, light and weak) Run2 (tested by Dragovich,1996)
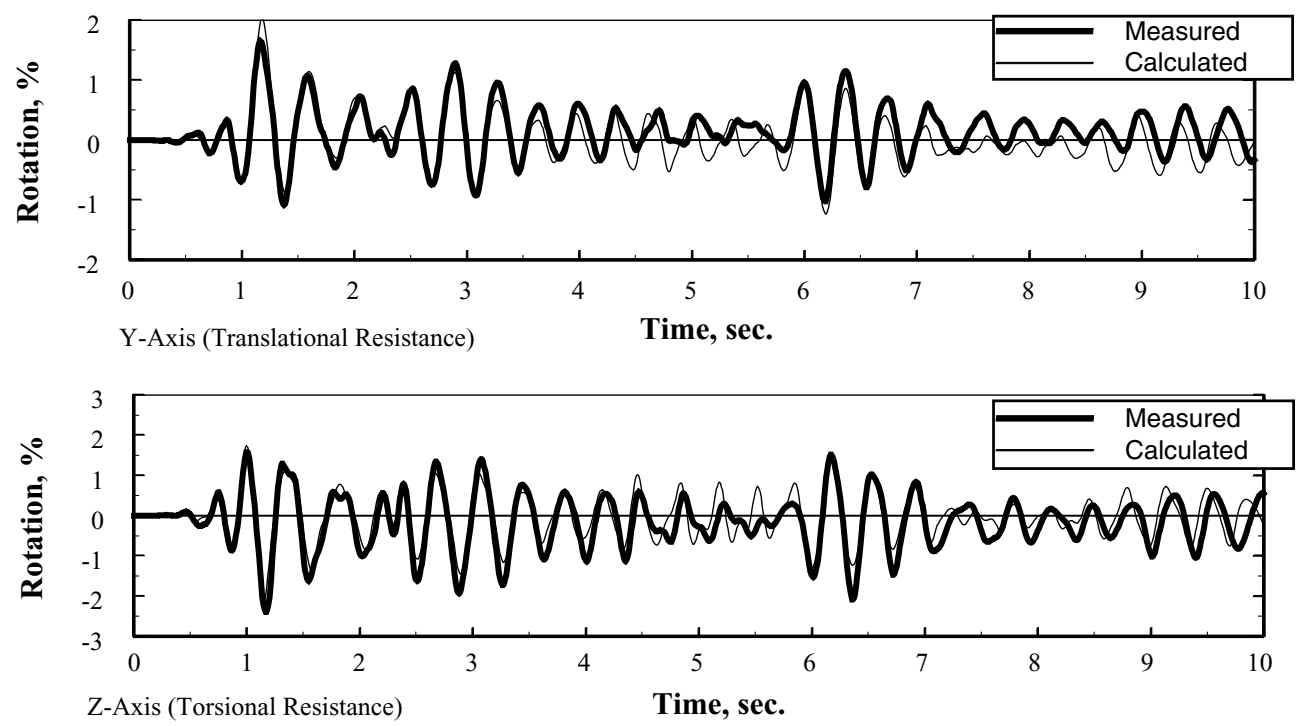

b) Displacement Histories for Rotation About Y and Z Axes,

Specimen ULS (uncoupled, light and strong) Run2 (tested by Dragovich,1996)

Fig. 10. Comparisons of the measured and the calculated rotation histories for Dragovich's tests (a) materially coupled,

(b) materially uncoupled.

The biaxial-moment traces of measured and calculated responses of a coupled specimen are presented in Figure 11. The loci of the measured (thin solid line) and calculated (thin broken lines) curves fall in the same regions. Both traces are seen to move along an axis from the 2 nd quadrant to the 4 th quadrant. The calculated limiting moment-envelope curves for the coupled specimens are also shown in the figure. Two momentenvelope curves are shown, one representing the yield of the first layer of bars and the other representing the yield of the second layer of bars in tension. For all specimens, the measured moments exceeded the calculated moment-envelope curves. The difference was less than $15 \%$. The excess moments were primarily attributed to the strain hardening and to the strain-rate effects.

There is an interesting and revealing aspect of the traces in Figure 11 showing the interaction of moments in two orthogonal directions. As the measured moment trace reaches the moment limit for the Y-axis at the lower-right or upper-left parts of the moment envelope 


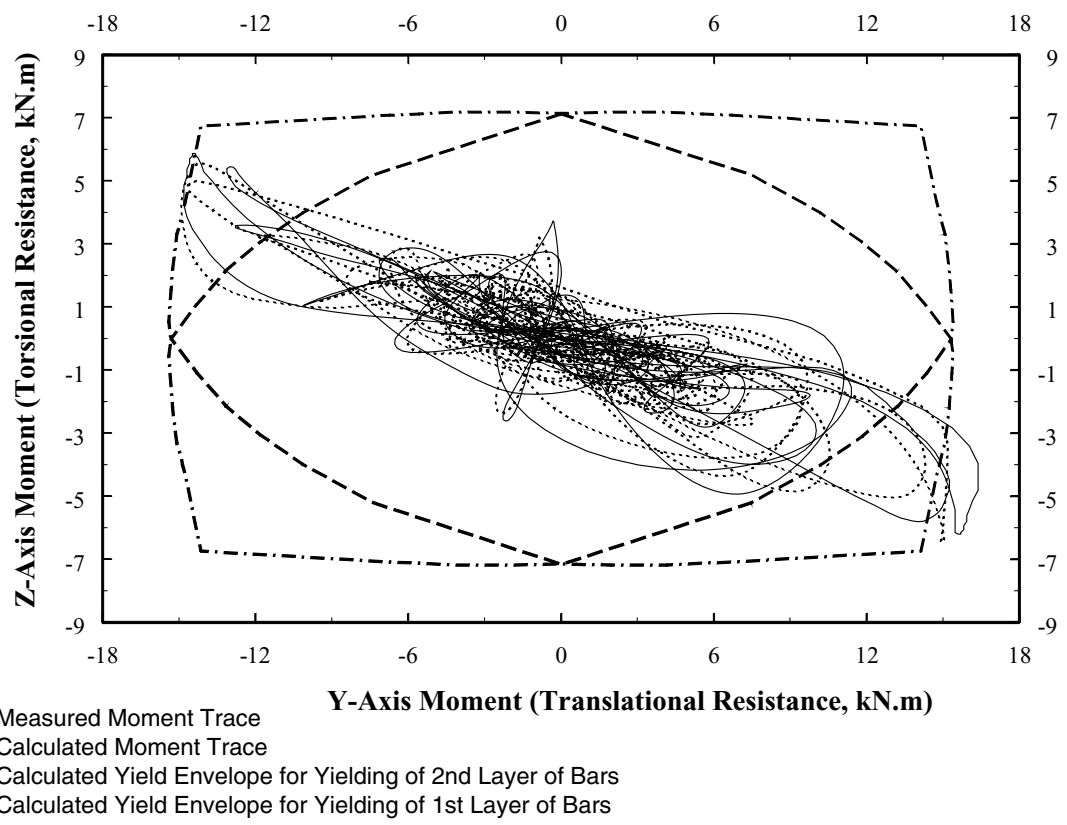

Fig. 11. Biaxial moment traces of the measured and the calculated responses of a coupled specimen and the calculated yield envelopes.

curves, it is observed that the Y-axis moment stays essentially the same but the $\mathrm{Z}$-axis moment increases. The calculated traces reproduce the same response. Accordingly, the "yield criterion" for biaxial moment is observed to be better reproduced by a "rectangular yield criterion" rather than by one having the shape of a rhomboid implied by yielding of the first level of bars.

It is also important to note that, in the measured responses of biaxially excited test specimens, two kinds of "pinching" were observed. The first kind was the type usually observed in uniaxially loaded elements related to slip or crack closing (Dragovich, 1996). The second kind was due to the biaxial nature of the loading. If the element had already yielded in direction-1, it yielded in direction- 2 at a lower moment but could regain strength in direction-2, if the element was unloaded in direction-1. As a result, slope of the moment-rotation response could change from steep to nearly flat, back to steep (Figures 12c and d), creating the effect which has been described as "pinching." The proposed algorithm is sensitive to the pinching caused by material interaction and provided a rationale for understanding the different sources of "pinching" in hysteresis loops.

\section{SUMMARY AND CONCLUSIONS}

An algorithm was developed to determine the response of reinforced concrete members subjected to biaxial

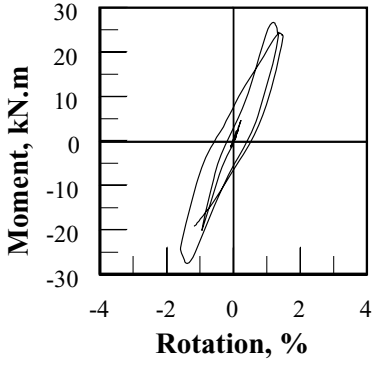

a) Y-axis

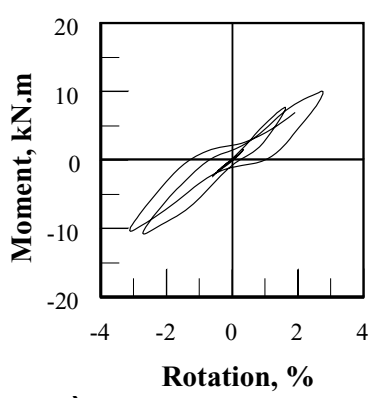

c) Z-axis

Measured

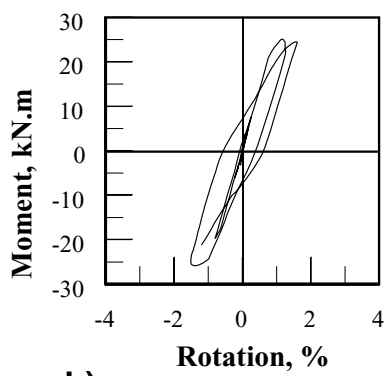

b) Y-axis

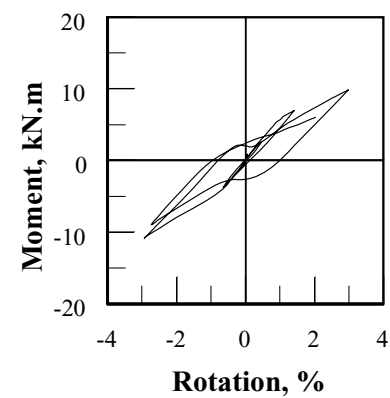

d) Z-axis

Calculated
Fig. 12. Measured and the calculated moment rotation hysteresis (first two seconds) of Specimen CHS (coupled, heavy, and strong), (a) and (b) Y-axis, (c) and (d) Z-axis.

ground excitation. The algorithm was based on nonlinear hysteretic response of concrete and steel in terms of their unit stress-strain properties and was tested using data from static and dynamic tests with biaxial loading. 
In order to establish the sensitivity of the proposed model to changes in input parameters under dynamic loading, a sensitivity study was performed. The sensitivity of the calculated results to changes in parameters defining reinforcement was strong for maximum-displacement, waveform, and periodicity. The effect of the parameters defining concrete was weak and limited to response at very low amplitudes. The choices for reinforcing steel parameters have to be considered carefully in assembling a model for calculating response.

Otani and Cheung's (1981) data were used to test the proposed hysteresis rules. Peak-force levels, mean stiffnesses, and hysteresis-loop areas were compared. The calculated maximum force levels were within $\pm 10 \%$ of the measured values. At drift ratios beyond yield, calculated energy dissipation (area of the loops) for each specimen was within $\pm 15 \%$ of the measured value and the calculated mean stiffnesses were within $\pm 10 \%$ of the measured values.

Dragovich's (1996) data were used to test the algorithm in the dynamic environment and also to study the effects of the combined torsional-translational loading in reinforced concrete. Maximum rotation, rotation-history, and the moment-rotation histories were studied. The mean ratio of the measured to calculated absolute-maximum rotations for the materially coupled specimens was 0.96 and for the materially uncoupled specimens, the mean ratio was 0.90 . Rotation histories of the specimens exhibited phases of low and high response. Agreement of the measured and the calculated curves was highly satisfactory for high response phases. For the low response phases, the agreement was not as good but satisfactory. The mean ratio of the measured to calculated absolute-maximum moments was 1.05 for the materially coupled and 1.12 for the materially uncoupled specimens. The difference between the measured and the calculated values was attributed to strain hardening and the strain-rate effects of the reinforcing bar.

The proposed algorithm provided a rationale for understanding two aspects of behavior under biaxial loading:

- The limits of the yield envelope in the two orthogonal directions remain essentially constant but the shape of the envelope changes from a near rhomboid, at first yield, to a rectangle, when all the tension reinforcement yields.

- In the tests, two types of "pinching" of the moment rotation response were observed. Calculations revealed that the first is related to concrete cracking and reinforcement slip as it has been observed under uniaxial bending and the second is due to interaction of moments in orthogonal directions under biaxial bending.

Comparisons of the calculated and measured results confirmed that the proposed algorithm was successful not only in providing excellent estimates of the maximum force and displacement responses, a result which has been achieved before, but also in leading to very good estimates of the behavior of the biaxially loaded reinforced concrete systems in all ranges of response. Considering that the elements of the model are explicit and simulate observed behavior of the materials in the stress-strain domain, it is concluded that the proposed algorithm provides a reliable basis for analytical assessment of the biaxial deflection/rotation response of reinforced concrete structures.

\section{ACKNOWLEDGMENTS}

The work presented in this article was supported by the funds from Izmir Institute of Technology and the Higher Education Council (YÖK) of Turkey. The study was performed at Purdue University with support from the National Science Foundation. The writers would like to acknowledge the support of these institutions. The writers would also like to acknowledge Dr. S. Otani and V. W. Cheung (Tokyo University) and Dr. J. Dragovich (Seattle University) for providing details of their valuable test data for the study.

\section{REFERENCES}

Aktan, A. E., Karlson, B. I. \& Sözen, M. A. (1973), Stress-Strain Relationships of Reinforcing Bars Subjected to Large Strain Reversals, Civil Eng. Studies, Structural Research Series No. 397, University of Illinois, Urbana-Champaign.

California Institute of Technology (1971), Earthquake Engineering Research Laboratory, Strong Motion Earthquake Accelerograms, Digitized and Plotted Data, Vol. II-Corrected Accelerograms and Integrated Ground Velocity and Displacement Curves, EERL, 71-50.

Dragovich, J. J. (1996), An experimental study of torsional response of reinforced concrete structures to earthquake excitation. Ph.D. thesis, University of Illinois, UrbanaChampaign.

Dönmez, C. (1998), A numerical model to simulate the behavior of reinforced concrete members subjected to biaxial earthquake excitation. Ph.D. thesis, Purdue University, W. Lafayette.

Hognestad, E. (1951), A Study of Combined Bending and Axial Load in Reinforced Concrete Members, Bulletin Series No. 399, University of Illinois Engineering Experiment Station, University of Illinois, Urbana-Champaign.

Karsan, I. D. \& Jirsa, J. O. (1969), Behavior of concrete under compressive loadings, ASCE Journal of the Structural Division, 95(12), 2543-63.

Menegotto, M. \& Pinto, P. E. (1973), Method of analysis for cyclically loaded R. C. Frames including changes in geometry 
and non-elastic behavior of elements under combined normal force and bending, IABSE Symposium on the Resistance and Ultimate Deformability of Structures Acted on by WellDefined Repeated Loads, pp. 15-22, Lisbon.

Otani, S. \& Cheung, V. W. (1981), Behavior of reinforced concrete columns under biaxial lateral load reversals, Publication 81-02, University of Toronto, Department of Civil Engineering.

Pujol, S. (2002), Drift capacity of reinforced concrete columns subjected to displacement reversals, Ph.D. thesis, Purdue University, W. Lafayette.

Ricles, M., Yang, S. Y. \& Priestley, M. J. N. (1998), Modeling nonductile $\mathrm{R} / \mathrm{C}$ columns for seismic analysis of bridges, ASCE Journal of Structural Engineering, 124(4), 415-25.

Sfakianis, M. G. \& Fardis, M. N. (1991), R/C column model for inelastic seismic response analysis in 3D, ASCE Journal of Engineering Mechanics, 117(12), 2270-87.

Stanton, J. \& McNiven, H. D. (1979), The development of a mathematical model to predict the flexural response of reinforced concrete beams to cyclic loads, using system identification, Report No: EERC 79/02, University of California, Berkeley.

Zeris, A. (1986), Three dimensional nonlinear response of reinforced concrete buildings, Ph.D. thesis, University of California, Berkeley.

\section{APPENDIX A}

\section{A.1. Concrete hysteresis model}

The model for concrete hysteresis is illustrated in Figure 1c. The limits of the compressive stress response are bounded by Ritter parabola up to the peak stress and a straight line joining the peak stress coordinates to a point on the strain axis (Hognestad, 1951). The rules for the bounds to the stress-strain curve for concrete are:

$$
\begin{array}{rlrl}
f_{c} & =0.0 & \varepsilon_{c}<\varepsilon_{t} \\
f_{c}=E_{c} \varepsilon_{c} & \varepsilon_{t} \leq \varepsilon_{c} \leq 0.0 \\
f_{c}=f_{c}^{\prime}\left[2 \frac{\varepsilon_{c}}{\varepsilon_{c o}}-\left(\frac{\varepsilon_{c}}{\varepsilon_{c o}}\right)^{2}\right] & 0.0<\varepsilon_{c} \leq \varepsilon_{c o} \\
f_{c}=f_{c}^{\prime}\left[1-\Omega\left(\varepsilon_{c}-\varepsilon_{c o}\right)\right] & \varepsilon_{c o}<\varepsilon_{c} \leq \varepsilon_{c x} \\
\varepsilon_{c x}=7 \varepsilon_{c o}, \quad \Omega=\frac{1}{\left(6 \varepsilon_{c o}\right)} & \\
f_{c}=0.0 & \varepsilon_{c x}<\varepsilon_{c}
\end{array}
$$

where

$$
\varepsilon_{t}=\frac{f_{t}}{E_{c}}, \quad \varepsilon_{c o}=\left(\frac{C_{1} f_{c}^{\prime}}{E_{c}}\right)
$$

$\varepsilon_{t}$ is the tensile strain corresponding to maximum tensile stress. $\varepsilon_{c o}$ is compressive strain of concrete corresponding to the compressive strength, $f_{c}^{\prime} . C_{1}$ is a constant defined to be 2.0 based on experimental data.
Equation (A3) defines the second-degree curve approximation to the concrete stress-strain curve proposed by Ritter (Hognestad, 1951). Equation (A4) defines the variation of the compressive stress capacity of unconfined concrete beyond the strain, $\varepsilon_{c o}$. The rules for cyclic loading are also illustrated in Figure 1c. Unloading and loading within the envelope curve follows a straight line, connecting the unloading point on the envelope $\left(\varepsilon_{c e n}\right.$, $\left.f_{c e n}\right)$ and the loading point $\left(\varepsilon_{c p}, 0.0\right)$. The strain $\varepsilon_{c p}$ that corresponds to the unloading point is computed from:

$$
\varepsilon_{c p}=\varepsilon_{c o}\left[\frac{1}{7}\left(\frac{\varepsilon_{c e n}}{\varepsilon_{c o}}\right)^{2}+\frac{1}{8} \frac{\varepsilon_{c e n}}{\varepsilon_{c o}}\right]
$$

Equation (A6) is a slightly simplified version of the unloading rule proposed by Karsan and Jirsa (1969). The unloading from the envelope curve is illustrated by line $\mathrm{CF}$ in Figure 1c. If the unloading continues and the concrete has not been previously cracked, travel follows line FH with a slope equal to $E_{c}$ to position $\mathrm{H}$ defined by the tensile strength of the concrete. Further travel will drop the stress to zero to follow path HLG. If the concrete is previously cracked, the unloading path follows FLG. Loading follows the path used in the most recent unloading. However, if the concrete is cracked, the loading path is GLFC. If loading continues beyond the last position on the envelope $\left(\varepsilon_{c e n}, f_{c e n}\right)$, the loading follows the envelope curve. If compressive strain goes beyond $\varepsilon_{c x}$, concrete stress becomes zero.

The concrete surfaces bounding a crack, on being compressed together, may not have a perfect fit. Consequently, raised portions on each bounding surface are likely to make contact before the crack closes completely. Compression is developed before the strain, in this case at point $F$, is reached. The path connecting $G$ to $\mathrm{E}$ in Figure 1c represents the early resistance gain of the concrete during crack closing. Recognizing that there is a range of "incomplete contact" before complete contact occurs, a set of hysteresis rules to simulate this phenomenon was selected. These rules are described in reference to Figure 1d. The detailed plot in Figure 1d shows a portion of the stress-strain curve for the concrete. Point $\mathrm{G}$ is defined at a strain $6 f_{t} / E_{c}$ from point $\mathrm{F}$, which is assumed to be the strain at zero stress for the most recent unloading from the envelope curve. If the calculated strain goes to a strain to the right of point $\mathrm{G}$, upon reversal, the stress-strain relationship follows path GE. The point $\mathrm{E}$ is located on the most recent unloading from the envelope curve at a stress of $f_{c e n} / 5$. If the stress reverses again before reaching point $\mathrm{E}$, unloading is assumed to occur along line GE.

If, after unloading to point $\mathrm{F}$, there is a stress reversal before reaching point $\mathrm{G}$, the loading curve makes a beeline for point E, as illustrated by line $\mathrm{G}^{\prime} \mathrm{E}$. Again, if 
there is another reversal within line $\mathrm{G}^{\prime} \mathrm{E}$, unloading occurs along line $\mathrm{G}^{\prime} \mathrm{E}$. If point $\mathrm{E}$ is reached during loading, unloading occurs along line $\mathrm{CF}$.

\section{A.2. Steel hysteresis model}

The selected steel-hysteresis model is a modification of the one developed by Stanton and McNiven (1979). It is a variable-parameter type model and based on the Menegotto-Pinto curves. A typical M-P curve is illustrated in Figure $1 \mathrm{~b}$ by the curve AB. The formulation is

$$
\sigma^{*}=b \varepsilon^{*}+\frac{(1-b) \varepsilon^{*}}{\left(1+\varepsilon^{* R}\right)^{\left(\frac{1}{R}\right)}}
$$

where

$$
\begin{gathered}
\varepsilon^{*}=\frac{\varepsilon-\varepsilon_{r}}{\varepsilon_{o}-\varepsilon_{r}}, \quad \sigma^{*}=\frac{\sigma-\sigma_{r}}{\sigma_{o}-\sigma_{r}} \\
b=\frac{E_{2}}{E_{1}}, \quad E_{1}=\frac{\sigma_{o}-\sigma_{r}}{\varepsilon_{o}-\varepsilon_{r}}
\end{gathered}
$$

$b$ is the ratio of the slopes of the primary curve and the asymptote. $E_{1}$ is the slope of the primary stiffness curve.

Before the first reversal, the results of the M-P model are similar to those of the bilinear steel model. If the fiber has not yielded in either direction, travel occurs along the primary curve (Figure 1b). After the first yield, in either direction, travel occurs along the secondary curve (Figure 1b). At the first unloading from the secondary curve, travel follows M-P curves. Primary curve, asymptote (secondary curve in Figure 1b) and the transition parameter $\mathrm{R}$ have to be defined for constructing an M-P curve. The primary curve and the asymptote need two points for their definitions, the point of reversal $\left(\varepsilon_{r}, \sigma_{r}\right)$ and the point of intersection for the primary curve and the asymptote $\left(\varepsilon_{o}, \sigma_{o}\right)$. The point of reversal is the unloading point. Consider the reversal point $\left(\varepsilon_{r}, \sigma_{r}\right)$ in Figure 1b. A tangent, with slope $\mathrm{E}_{1}$, is drawn at point $\left(\varepsilon_{r}, \sigma_{r}\right)$. This tangent crosses the strain axis at $\varepsilon_{z}$. The initial bilinear loading curve is shifted so that its origin is at point $\varepsilon_{z}$. The tangent drawn at point $\left(\varepsilon_{r}, \sigma_{r}\right)$ is extended to intersect with the shifted curve. The point of intersection $\left(\varepsilon_{o}, \sigma_{o}\right)$ becomes the new breakpoint for defining travel in that direction. A new set of bounding curves has to be defined at every reversal.

Values of $E_{1}$ and $R$ are defined by Stanton and McNiven (1979) based on experimental data. The stiffness $E_{1}$, is modified to have an initial modulus of $200,000 \mathrm{MPa}$,

$$
\begin{aligned}
E_{1}= & 200000-744000 \varepsilon_{r} \\
& -1400\left(1.0-\exp \left(-390.0 \varepsilon_{r}\right)\right), \quad \text { in } \mathrm{MPa} \\
E_{1} \geq & 152000, \text { in } \mathrm{MPa}
\end{aligned}
$$

Definition of the $R$ is modified by adding constant, $C_{2}$ to the original form and it is selected as 17 based on the experimental data obtained by Aktan (1973),

$$
R=\frac{r \sigma_{y}}{C_{2}}
$$

where, $\quad r=0.51-7.53 \varepsilon_{\max c}^{p}$ in tension,

$$
r=0.48-4.36 \varepsilon_{\max t}^{p} \text { in compression }
$$

There are several advantages of using the M-P curves. A model based on the M-P curves is computationally economical in this application, because stress is computed from strain. It is explicit. Distinct characteristics of the steel hysteresis can be captured with the parameters of the M-P curves. This permits the manipulation of the parameters independently. In addition, the parameters can be obtained conveniently from steel hysteresis tests (Stanton and McNiven, 1979).

\section{APPENDIX B: STEPS OF THE ITERATIVE PROCEDURE FOR CALCULATION OF MOMENT-DEFLECTION RELATIONSHIP}

Before starting the iteration cycle, the following parameters need to be available. These parameters are refreshed after reaching convergence in every step.

- Curvature $\phi_{Y}$ and $\phi_{Z}$ along axes $Y$ and $Z$ (Figure 2b).

- Moments $M_{Y}$ and $M_{Z}$ along axes $Y$ and $Z$ (Figure 2d).

- Length of the "yield region," $d_{y r}$ (Figure 2a).

- Displacements $d_{Y}$ and $d_{Z}$ along axes $Y$ and $Z$.

Step 1: Estimate incremental curvatures using the relation between incremental displacement and the curvature for a linearly elastic member.

$$
\Delta \phi_{Y}=\frac{3 \Delta d_{Z}}{L^{2}}, \quad \Delta \phi_{Z}=\frac{3 \Delta d_{Y}}{L^{2}}
$$

Step 2: Calculate the direction of curvature using the curvature values obtained by adding the calculated curvature increments to the values at the beginning of the step. The direction of deflection is orthogonal to the direction of the curvature (Figure 2b).

Step 3: Calculate the incremental moments (in the $Y$ and $Z$ directions, Figure $2 d$ ) on the section using the equilibrium of forces normal to the section.

Step 4: Calculate the new length for the "yield region" of the member using the incremental moments calculated in Step 3. The "yield region" length is calculated using the moment reached with the latest increments and the yield moment in the direction of the current moment. If the new length is larger than the current length refresh the length with the new value, otherwise retain the old value. 


$$
\begin{aligned}
M_{\text {calc }} & =\sqrt{\left(M_{Y}+\Delta M_{Y}\right)^{2}+\left(M_{Z}+\Delta M_{Z}\right)^{2}} \\
d_{y r} & =\frac{\left(M_{\text {calc }}-M_{Y \theta}\right)}{\left(\frac{M_{\text {calc }}}{L}\right)}
\end{aligned}
$$

The yield moment in the current direction is calculated by assigning the yield strain to the bar that is furthest from the neutral axis (Figure 2d). After obtaining the "yield region" length, separate it to its components along axes $Y$ and $Z, L_{y}$ and $L_{z}$.

Step 5: Calculate the moments at the end of the "yield region."

Step 6: Using the simplified moment-curvature relationships (observing that the dependence of the moment-curvature relation to the loading history is weak up to the yielding and can be simulated, a simplified relation is utilized as shown in Figure 2c) and the moment values obtained in the Step 5, calculate the curvatures at the end of the "yield region," $\phi_{Y 1}$ and $\phi_{Z 1}$.

Step 7: Calculate the flexural deflections, using the assumed curvature distributions obtained (Figure 2a).

Step 8: Calculate the deflection caused by the bar slip.

Step 9: Calculate the shear deflections.

Step 10: Add the deflection components calculated at Steps 7-9 to determine the total deflection. Incremental deflection is the difference between the deflection calculated and the deflection at the beginning of the increment.

$$
\begin{aligned}
& \left(\Delta d_{Y}\right)_{\text {calc }}=\left(d_{Y}\right)_{\text {calc }}-d_{Y} \\
& \left(\Delta d_{Z}\right)_{\text {calc }}=\left(d_{Z}\right)_{\text {calc }}-d_{Z}
\end{aligned}
$$

If the calculated deflection increments are within the convergence limit of the given deflection increments, the iteration process for the step is stopped. Otherwise, new curvature increments are estimated using the following formulation.

$$
\begin{aligned}
\left(\Delta \phi_{Y}\right)_{\text {new }} & =\frac{\Delta d_{Z}}{\frac{\left(\Delta d_{Z}\right)_{\text {calc }}}{\Delta \phi_{Y}}}, \\
\left(\Delta \phi_{Z}\right)_{\text {new }} & =\frac{\Delta d_{Y}}{\frac{\left(\Delta d_{Y}\right)_{\text {calc }}}{\Delta \phi_{Z}}}
\end{aligned}
$$

Steps 2-10 are repeated until acceptable convergence is reached. This procedure requires a series of iterations of assuming a curvature increment and arriving at an incremental displacement. The needed convergence is assumed to have been achieved, if the calculated deflection increment is within $0.0025 \mathrm{~cm}$. of the incremental deflection. For the time increments used, the typical displacement increment value in the nonlinear range is $0.2 \mathrm{~cm}$. The distribution of the curvature along the span is set by the magnitude of the assumed curvature. In successive iterations, the curvature changes and its distribution may also change. It may be noted that the numerical procedure outlined above give results that are independent of the step size. However, the number of iterations required to predict the correct curvatures and the neutral axis depth to satisfy the axial stress equilibrium increase with larger step size.

\section{APPENDIX C: NOTATION}

$C_{2}=17$, based on experimental data obtained by Aktan (1973).

$E_{2}=$ Slope of the asymptote (Figure 1b).

$E_{c}=$ Young's Modulus of the concrete.

$L=$ Length of the member.

$M_{\text {calc }}=$ Magnitude of calculated moment vector.

$M_{Y \theta}=$ Yield moment in the current direction.

$R=$ Parameter defining the transition of travel from the primary curve to the asymptote.

$\left(d_{Y}\right)_{\text {calc }}=$ Calculated deflection along the Y-axis. $d_{y r}=$ "Yield region" length.

$\left(d_{Z}\right)_{\text {calc }}=$ Calculated deflection along the Z-axis.

$f_{c}=$ Compressive stress of the concrete.

$f_{c}^{\prime}=$ Compressive strength of the concrete.

$f_{t}=$ Tensile strength of the concrete.

$\left(\Delta d_{Y}\right)_{\text {calc }}=$ Calculated deflection increment along the Y-axis.

$\left(\Delta d_{Z}\right)_{\text {calc }}=$ Calculated deflection increment along the $\mathrm{Z}$-axis.

$\Delta d_{Y}=$ Given incremental deflection at the free end of the member along Y-axis.

$\Delta d_{Z}=$ Given incremental deflection at the free end of the member along Z-axis.

$\Delta \phi_{Y}=$ Guess for the incremental curvature along Y-axis.

$\Delta \phi_{Z}=$ Guess for the incremental curvature along Z-axis.

$\Omega=$ Constant that defines the descending slope of the concrete stress-strain curve.

$\varepsilon_{c}=$ Concrete strain.

$\varepsilon_{c e n}=$ Position of the maximum excursion on the envelope curve (Figure 1c).

$\varepsilon_{c p}=$ Concrete strain on the strain axis for unloading from the position of maximum excursion (Figure 1c). 
$\varepsilon_{c x}=$ Maximum compressive strain of the concrete.

$\varepsilon_{o}, \sigma_{o}=$ Strain and stress coordinates at the intersection of the primary curve and the asymptote (Figure 1b).

$\varepsilon_{\text {maxc }}^{p}=$ The maximum plastic strain in a half loop, in compression.

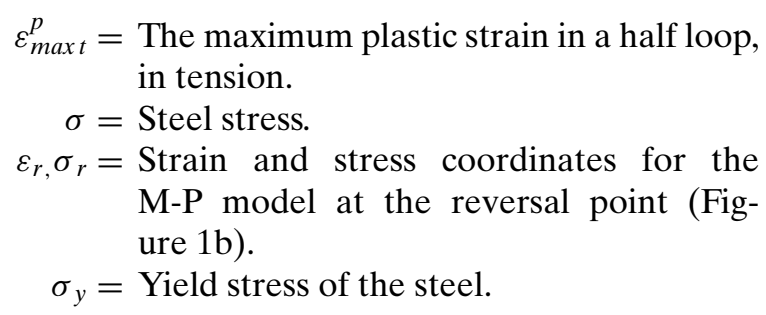

